\title{
High Order Momentum States by Light Wave Scattering
}

\author{
Xiaoji Zhou and Xuguang Yue \\ School of Electronics Engineering and Computer Science, \\ Peking University, Beijing \\ China
}

\section{Introduction}

Atomic interferometry is very useful in fundamental studies of coherence, decoherence and phase shifts and for practical precision measurements, with the example of gravimeters, gyroscopes, and gradiometers (Cronin et al., 2009). Bose-Einstein Condensate (BEC) based atomic interferometry provides high contrasts, long integration times and the possible use of small devices (Baumgärtner et al., 2010; Sapiro et al., 2009a; Torii et al., 2000). In such atomic interferometry, coherent momentum manipulation is very effective for splitting and recombining the condensate (Deng et al., 1999; Kozuma, Deng et al., 1999; Ovchinnikov et al., 1999), for realizing the interference. In some precision measurements, the accumulated phase is positively correlated to the atomic velocity, so that the larger the atomic momentum is, the more precise the measurements can be if the measuring time is unchanged.

Based on our previous experimental and theoretical work about the interaction between laser pulses and a BEC, in this chapter we mainly present methods of getting high order of momentum states by resonant superradiance (Zhou et al., 2009) and by multi-pulse Kapitza-Dirac scattering (Xiong et al., 2011), corresponding to travelling wave scattering and standing wave scattering, respectively.

In the superradiant Rayleigh scattering (Bar-Gill et al., 2007; Guo et al., 2008; Inouye et al., 1999; Kozuma, Suzuki et al., 1999; Moore \& Meystre, 1999; Pu et al., 2003; Sadler et al., 2007; Schneble et al., 2003; Slama et al., 2007; Zobay \& Nikolopoulos, 2006), the spatial and time evolutions of superradiant scattering are studied for a weak pump beam with different frequency components traveling along the long axis of an elongated Bose-Einstein condensate. Through the analysis of the mode competition between the different resonant channels and the local depletion of the spatial distribution, we can get a large number of high-order forward modes by resonant frequency components of the pump beam (Zhou et al., 2009).

On the other hand, the atomic diffraction from a standing wave light grating is a primary method of atomic momentum manipulation. Different to usual separation of the Kapitza-Dirac regime, the Bragg regime and the channeling regime (Keller et al., 1999), the scattering process is described by the projection of atomic states between the momentum presentation and Bloch states form by the scattering standing waves. According to this 
method, we are able to design and realize several specific high order momentum states for the atomic interferometry (Xiong et al., 2011).

\section{High-order momentum modes by resonant superradiant scattering}

A typical superradiance experiment consists in a far off-resonant laser pulse traveling along the short axis of a cigar-shaped BEC sample (Inouye et al., 1999), the scattered lights, called end-fire modes, propagate along the long axis of the condensate, and the recoiled atoms are refereed to as side modes. A series of experiments (Bar-Gill et al., 2007; Kozuma, Suzuki et al., 1999; Sadler et al., 2007; Schneble et al., 2003; Slama et al., 2007) have sparked related interests in phase-coherent amplification of matter waves (Kozuma, Suzuki et al., 1999; Schneble et al., 2003), quantum information (Bar-Gill et al., 2007), collective scattering instability (Slama et al., 2007), and coherent imaging (Sadler et al., 2007). Several theoretical descriptions of these cooperative scattering in BEC with single-frequency pump have also been presented (Guo et al., 2008; Moore \& Meystre, 1999; Pu et al., 2003; Zobay \& Nikolopoulos, 2006).

For the long and weak pump beam, we can observe the forward peaks correspond to Bragg diffraction of atoms (Inouye et al., 1999), where the high order scattering is limited by detuning barriers for the end-fire mode radiation (Zobay \& Nikolopoulos, 2007). On the other hand, a X-shaped recoiling pattern is demonstrated in a short and strong pulse as Kapitza-Dirac diffraction of atoms (Schneble et al., 2003), where an atom in the condensate absorbs a photon from the pump laser, then emits a photon into an end-fire mode, and recoils forwardly. Meanwhile another atom absorbs a photon from the end-fire modes, emits into the pump beam and finally recoils backwardly. In this case, there is an energy mismatch of four times the one-photon recoil kinetic energy $\hbar \omega_{r}$ in backward scattering, which then remains very weak unless a short pumping pulse with a broad spectrum is used. Hence, two phase-locked incident lasers with the frequency difference $\Delta \omega$ compensating for the energy mismatch has been used (Bar-Gill et al., 2007; Cola et al., 2009; Yang et al., 2008), which is named resonant superradiance, where a large number of backward recoiling atoms can be produced.

Followed that, it is attractive to extent this idea to achieve a high momentum transfer by overcoming the detuning barriers, by a weak and long pump beams with the resonant frequency. It requires to analysis the competition between the different transition channels and the spatial distribution of different modes. Because the above traditional superradiant-scattering configuration involves many atomic side modes coupled together, to simplify it, we chose another configuration where a pump beam travels along the long axis of the BEC. This scheme is widely studied in photon echo (Piovella et al., 2003), decoherence (Fallani et al., 2005), spatial distribution effects (Li, Zhou et al., 2008) and self-organized formation of dynamic gratings (Hilliard et al., 2008). Since the pulse length is far longer than the initial spontaneous process (Zobay \& Nikolopoulos, 2006), we choose the semi-classical theory which can well describe the experimental results (Bar-Gill et al., 2007; Yang et al., 2008; Zobay \& Nikolopoulos, 2006).

In this section, we first introduce the semi-classical theory for the superradiance scattering with a several-frequency pump in the weak coupling. Then the spatial and time evolutions of scattered modes are analyzed for two-frequency pump beam, we find the backward first 


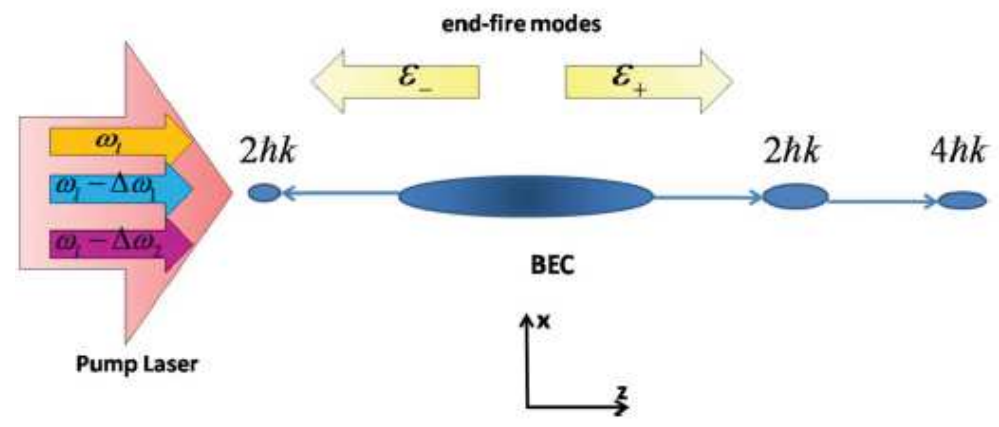

Fig. 1. (Color online) Our experimental scheme. A cigar-shape BEC is illuminated by a far off-resonant laser pulse along its long axis $\hat{\mathbf{z}}$. Collective Rayleigh scattering induces superradiance. Two end-fire modes, which are also along $\hat{\mathbf{z}}$ axis, form in superradiance process and the 1st-order recoiled atoms obtain a momentum of $2 \hbar \mathbf{k}$.

order scattering mode is suppressed at the resonant condition $\Delta \omega=8 \omega_{r}$ and the forward second order mode is enhanced, resulting from the combination of mode competition effects and spatial distribution of the modes. The case of the three-frequency pump beams for a large number of the forward third order scattering modes, and the higher modes for more resonant frequencies are studied, which supplies a new method to get a large number of atoms in higher order forward modes. Finally, some discussion and conclusion are given.

\subsection{Model for a multiple-frequency end-pumped beam}

We consider the pump laser, with amplitude $\mathcal{E}_{l}(t)$, polarization $\mathbf{e}_{\mathbf{y}}$, wave vector $\mathbf{k}_{l}$, frequencies $\omega_{l}$ and $\omega_{l}-\Delta \omega_{n}$, propagating along the long axis $\hat{\mathbf{z}}$ of an elongated BEC, $\mathbf{E}_{l}=\mathcal{E}_{l}(t) \mathbf{e}_{\mathbf{y}}[(1+$ $\left.\Sigma_{n} e^{i \Delta \omega_{n} t}\right) e^{i\left(k_{l} z-\omega_{l} t\right)}+$ c.c. $] / 2$, as shown in Fig. 1. When supperradiant Rayleigh scattering happens, end-fire modes spread along the same axis. The $\mathcal{E}_{+}$mode has the same direction as the incident light and mainly interacts with the right part of the condensate, and the $\mathcal{E}_{-}$ mode overlaps with the left part of the condensate. The atoms are recoiled to some discrete momentum states with momentum $2 m \hbar \mathbf{k}$, where $m$ is an integer and the wave vector of end-fire mode light $k$ is approximated as $k_{l}$ for energy conservation. The total electric field $\mathbf{E}(\mathbf{r}, t)=\mathbf{E}^{(+)}+\mathbf{E}^{(-)}$is given by (Bar-Gill et al., 2007; Li, Zhou et al., 2008; Yang et al., 2008; Zobay \& Nikolopoulos, 2006)

$$
\mathbf{E}^{(+)}(\mathbf{r}, t)=\left[\left(1+\sum_{n} e^{i \Delta \omega_{n} t}\right) \mathcal{E}_{l}(t) e^{-\mathrm{i}\left(\omega_{l} t-k_{l} z\right)} / 2+\mathcal{E}_{-}(z, t) e^{-\mathrm{i}(\omega t+k z)}\right] \mathbf{e}_{\mathbf{y}}
$$

where $\omega=c k, \mathbf{E}^{(-)}=\mathbf{E}^{(+) *}$, and $\mathcal{E}_{+}$is ignored because it has the same wave vector as the pump beam but is very small in comparison to $\mathcal{E}_{l}$. $\Delta \omega_{n}$ satisfies the condition $\Delta \omega_{n} \ll \omega_{l}$ (Bar-Gill et al., 2007) and the initial phases of the different frequency components are assumed to be zero.

Since the BEC is tightly constrained in its short axis $(\hat{\mathbf{x}}, \hat{\mathbf{y}})$ in the present superradiance setup and the Fresnel number of the optical field is around 1, one dimensional approximation is usually used (Bar-Gill et al., 2007; Hilliard et al., 2008; Inouye et al., 1999; Li, Zhou et al., 
2008). We expand the wavefunction of the condensate $\psi(\mathbf{r}, t)$ in momentum space, $\psi(\mathbf{r}, t)=$ $\sum_{m} \phi_{m}(z, t) \times e^{-i\left(\omega_{m} t-2 m k z\right)}$, where $\phi_{m}(z, t)=\psi_{m}(z, t) / \sqrt{A}, \omega_{m}=2 \hbar m^{2} k^{2} / M$ with $M$ the atomic mass, $m=0$ corresponds to the residual condensates, $m \neq 0$ denotes the side modes, and $A$ is the average cross area of the condensate perpendicular to $\hat{\mathbf{z}}$. Using the Maxwell-Schrödinger equations, we obtain dynamics equations for $\phi_{m}(z, t)$,

$$
\mathrm{i} \frac{\partial \phi_{m}}{\partial t}=-\frac{\hbar}{2 M} \frac{\partial^{2} \phi_{m}}{\partial z^{2}}-\frac{2 \mathrm{i} m \hbar k}{M} \frac{\partial \phi_{m}}{\partial z}+\bar{g}\left[\mathcal{E}_{-}^{*} \phi_{m-1} e^{-4 \mathrm{i}(1-2 m) \omega_{r} t}+\mathcal{E}_{-} \phi_{m+1} e^{-4 \mathrm{i}(1+2 m) \omega_{r} t}\right],
$$

where $\omega_{r}=\hbar k_{l}^{2} / 2 M$ is the recoil frequency, the coupling between modes is given by

$$
\bar{g}(t)=g\left(1+\sum_{n} e^{i \Delta \omega_{n} t}\right),
$$

with the coupling factor $g=\sqrt{3 \pi c^{3} R /\left(2 \omega_{l}^{2} A L\right)}, R$ is the Rayleigh scattering rate of the pump components, and $L$ is the BEC length.

The first term on the right-hand-side of Eq.(2) describes the dispersion of $\phi_{m}$, and the second term gives rise to their translation. The terms in square brackets describe the atom exchange between $\phi_{m}$ and $\phi_{m+1}$ or $\phi_{m-1}$ through the pump laser and end-fire mode fields. An atom in mode $m$ may absorb a laser photon and emit it into end-fire mode $\mathcal{E}_{-}$, and the accompanying recoil drives the atom into $m+1$ mode, hence atoms with mode $m+1$ can emerge in forward scattering. On the other hand, in the backward scattering, atoms with mode $m$ absorb one $\mathcal{E}_{-}$ mode photon, deposit it into the laser mode and go into mode $m-1$. The envelope function of end-fire mode $\mathcal{E}_{-}$is given by

$$
\mathcal{E}_{-}=-\mathrm{i} \frac{\omega_{r} \bar{g}}{2 c \varepsilon_{0}} \int_{z}^{+\infty} \mathrm{d} z^{\prime} \sum_{m} \phi_{m}\left(z^{\prime}, t\right) \phi_{m+1}^{*}\left(z^{\prime}, t\right) e^{\mathrm{i} 4(2 m+1) \omega_{r} t}
$$

indicating that the end-fire mode field $\mathcal{E}_{-}$is due to the transition between $m$ and $m+1$ mode and the magnitude of $\mathcal{E}_{-}$depends on the spatial overlap between the two modes. In addition, there is a frequency difference of $8 \omega_{r}$ between adjacent modes.

\subsection{Mode competition for a two-frequency pump beam}

In the case of a single-frequency pump in the weak coupling regime, the evolution of the side modes and the end-fire mode indicates that the scattering is a localized process. For this end-pumping configuration, the scattering first starts on the leading edge of the BEC and then moves towards the tailing edge. To investigate the effect of the two-frequency pump beam, the different frequency components of the end-fire mode which indicate the energy change during the scattering are depicted in Fig. 2. The momentum of side mode $m=n$ is $2 n \hbar \mathbf{k}$, and its kinetic energy is $4 n^{2} \hbar^{2} \mathbf{k}^{2} / 2 M=4 n^{2} \hbar \omega_{r}$. For the pump component with frequency $\omega_{l}$, atoms from the condensate are pumped to the side mode $m=1$ and emit end-fire mode photons with frequency $\omega_{l}-4 \omega_{r}$ spontaneously. However, in the backward scattering process, an atom in the condensate absorbs the end-fire mode $\left(\omega_{l}-4 \omega_{r}\right)$ and emits a photon with frequency $\omega_{l}$ back into the pump laser. Since energy is not conserved in backward-scattering, the backward side mode is not populated in weak-pulse regime. Side mode $m=2$ is also not populated 

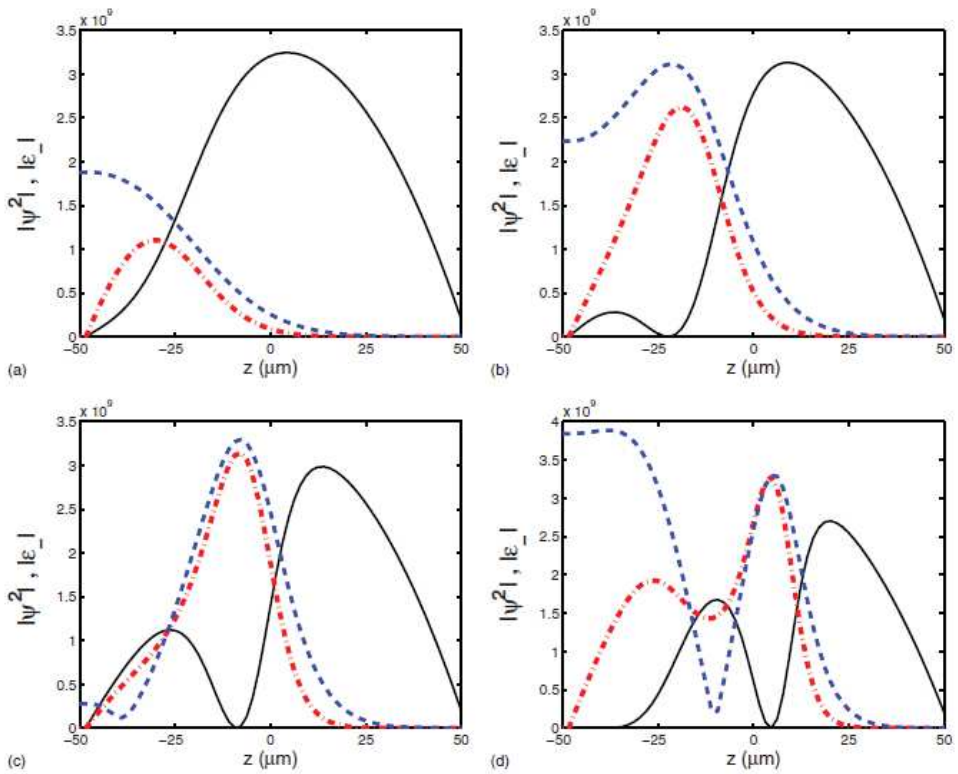

Fig. 2. (Color online) Light-field components of a two-frequency pump laser. The broad arrows are the pump laser and narrow ones are the end-fire mode (scattering optical field). In a spontaneous process, atoms in the condensate absorb photons from the pump laser with frequencies $\omega_{l}$ and $\omega_{l}-8 \omega_{r}$, are scattered to side mode $m=1$ and emit end-fire mode photons with frequency $\omega_{l}-4 \omega_{r}$ (dashed arrow) and $\omega_{l}-12 \omega_{r}$ (dotted arrow), respectively. Meanwhile, atoms in the condensate can also absorb end-fire mode photons with frequency $\omega_{l}-4 \omega_{r}$, be scattered back to side mode $m=-1$ and emit photons with frequency $\omega_{l}-8 \omega_{r}$ (solid arrow), resonating to one of the pump laser components. The side mode $m=1$ can absorb pump laser photons with frequency $\omega_{l}$ and be scattered to mode $m=2$, emitting photons with frequency $\omega_{l}-12 \omega_{r}$ resonating to the existing end-fire mode.

due to the energy barrier. However, if we use the two components pump laser with frequency difference $8 \omega_{r}$, i.e. resonant frequency difference, the energy mismatch can be compensated by the pump laser.

Although the resonant condition for the backward mode is satisfied, it should be noticed that two scattering channels exist almost simultaneously. One is atoms scattered from side mode $m=0$ to $m=-1$ and the other is from $m=1$ to $m=2$, resulting in mode competition. The transition from mode $m=1$ to $m=2$ requires absorbtion of photons from pump laser, while the backward transition takes photons from the end-fire mode. Because the intensity of the pump laser is far greater than that of the end-fire mode, the transition from $m=1$ to $m=2$ has a bigger probability than the transition from $m=0$ to $m=-1$. Thus the population of the backward mode $m=-1$ is suppressed even at the resonant condition, while the forward mode $m=2$ is enhanced.

However, the existence of competition between these two channels may not lead to the suppression of the backward mode. If these two channels happen in different spacial parts 

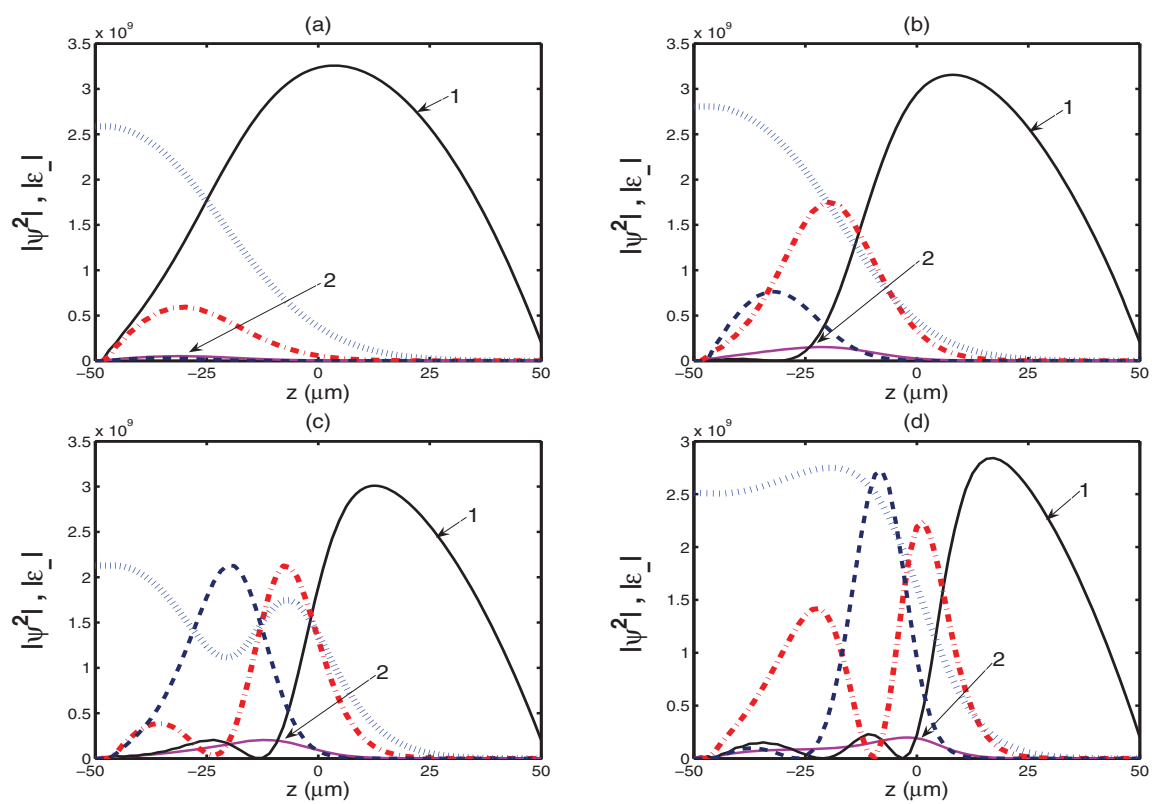

Fig. 3. (Color online) Spatial distribution of the side modes $\left|\psi^{2}\right|$ and the end-fire mode $\left|\varepsilon_{-}\right|$in the weak coupling regime $\left(g=1.25 \times 10^{6} s^{-1}\right)$ with the two-frequency pump for different pulse durations: $150 \mu s$ (a); $200 \mu s$ (b); $250 \mu s$ (c); $300 \mu s$ (d). Condensate mode $m=0$ is the solid line-1, backward first-order side mode $m=-1$ is the solid line-2, forward first-order side mode $m=1$ is the dash-dotted line, forward second-order side mode $m=2$ is the dashed line, and end-fire mode is the dotted line.

of the condensate, then both of side mode $m=-1$ and $m=2$ will be enhanced. The suppression of backward mode $m=-1$ and the enhancement of mode $m=2$ need that these two scattering channels happen in the same area. Therefore, the spatial distribution effect should be considered.

We analyze the spatial effect when second-order forward side mode and backward side mode are populated at the resonant condition $\Delta \omega=8 \omega_{r}$. The evolution of spatial distribution of side modes and end-fire mode is shown in Fig.3. Superradiance first starts on the leading edge of the BEC, as shown in Fig.3(a). Although the backward first-order side mode $m=-1$ is populated through the overlap between end-fire mode $\mathcal{E}_{-}$and side mode $m=0$, it is very small and emerges at the leading-edge of the BEC. Since the overlap between end-fire mode and side mode $m=1$ is in the same area, the population of side mode $m=2$ is obvious on this edge, as shown in Fig.3(b). Side mode $m=2$ grows more rapidly than side mode $m=-1$, which means more atoms are scattered from side mode $m=1$ to $m=2$ than that from $m=0$ to $m=-1$.Then the first peaks of side modes $m=1$ and $m=2$ move to the center of the BEC, as shown in Fig.3(c). Though the movement of the peaks is similar to that in the case of a single-frequency pump laser, one major difference is that the regrowth of side mode $m=0$ is very little, hence nearly all the atoms on this edge are forwardly scattered. Due to the nearly-complete depletion of the condensate, atoms are mainly transferred between side 

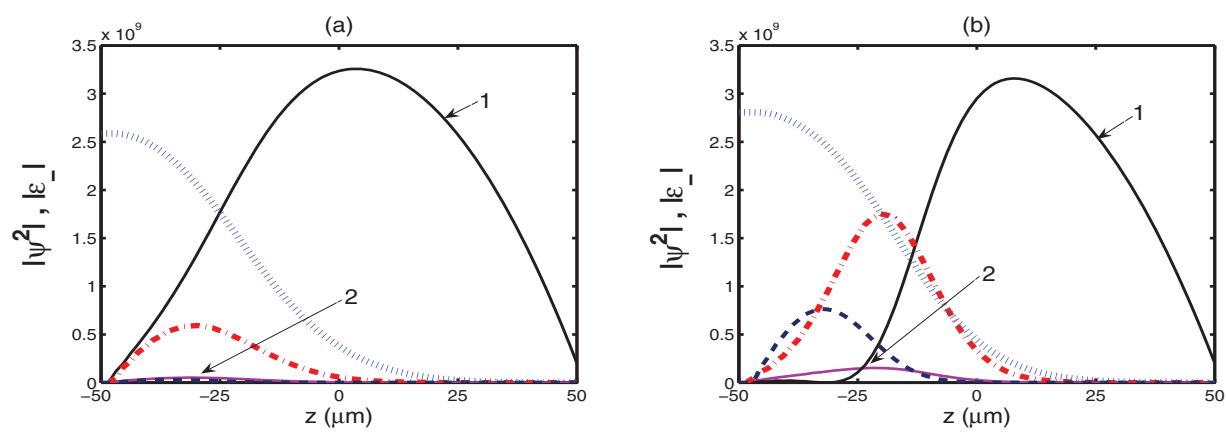

Fig. 4. (Color online) Normalized side mode populations versus time: (a) for a single-frequency pump beam; (b) for a two-frequency resonant pump beam. In both cases the coupling constant is kept $g=1.55 \times 10^{6} \mathrm{~s}^{-1}$. The side mode are: $\mathrm{m}=-1$ (solid); $\mathrm{m}=1$ (dotted); $\mathrm{m}=2$ (dashed).
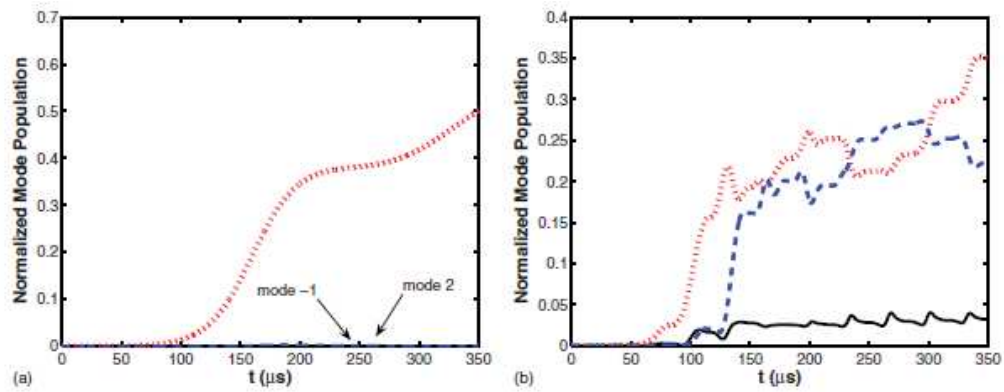

Fig. 5. (Color online) The light-field components of a three-frequency pump laser. The broad arrows are the pump laser and narrow ones are the end-fire mode.

mode $m=1$ and $m=2$. The apparent regrowth of side mode $m=1$ on the leading-edge shown in Fig.3(d) indicates that there are Rabi oscillations between side modes $m=1$ and $m=2$ in the depleted area of the condensate.

The time evolution of several side modes populations normalized by the total atom number are depicted by Fig.4. Fig.4 (a) shows that using a single-frequency pump laser cannot produce backward mode $m=-1$ or forward higher mode $m=2$ in the weak-pulse regime. Using a resonant two-frequency pump beam with the same intensity, modes $m=-1$ and $m=2$ increased, as shown in Fig.4 (b), however, the forward mode is greatly enhanced while the backward mode remains very small.

\subsection{The third order forward modes enhanced with a three-frequency pump beam}

The second forward side mode $m=2$ is greatly enhanced with a resonant two-frequency pump beam, however, the populations of higher forward modes such as $m=3$ are very small as the channel from the second forward mode to the third forward mode is not resonant with the exiting optical field. To get a large number mode for $m=3$, Fig.5 depicts the scheme 
(a)

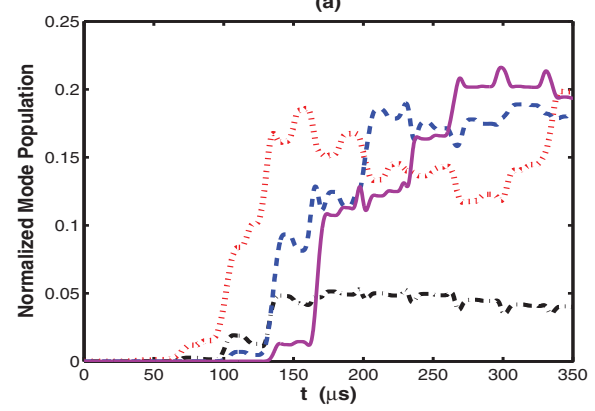

(b)

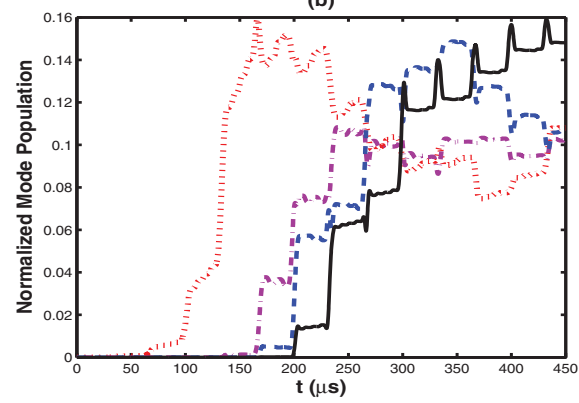

Fig. 6. (Color online) Normalized side mode populations versus time with the coupling constant $g=1.55 \times 10^{6} \mathrm{~s}^{-1}$ : (a) for a three-frequency pump laser: $m=-1$ (dash-dotted), $m=1$ (dotted), $m=2$ (dashed), $m=3$ (solid) ; (b) for a five-frequency pump laser: $m=1$ (dotted), $m=3$ (dash-dotted), $m=4$ (dashed), $m=5$ (solid) .

of the three-frequency pump beam with the frequencies of the pump laser $\omega_{l}, \omega_{l}-8 \omega_{r}$ and $\omega_{l}-16 \omega_{r}$. The frequency components $\omega_{l}, \omega_{l}-8 \omega_{r}$ and $\omega_{l}-16 \omega_{r}$ both have the resonant frequency difference. Hence, there could be two channels to form the backward side mode $m=-1$ but the enhancement of the backward scattering is small because of the formation of higher forward side modes. There are also two channels to form side mode $m=2$. One thing different from the two-frequency pump beam is that there is also a channel to form side mode $m=3$ for the reason that atoms in side mode $m=2$ absorb pump laser photons with frequency $\omega_{l}$, are then scattered to mode $m=3$ and eventually emit end-fire mode photons with frequency $\omega_{l}-20 \omega_{r}$ which is resonant to an existing end-fire mode. This means that more atoms in side mode $m=2$ will be pumped to side mode $m=3$ and less will be transferred back to side mode $m=1$, a competition between side mode $m=3$ and $m=1$ is set up. As a result, side mode $m=3$ will be enhanced and $m=1$ will be reduced relatively.

Fig.6(a) is the simulated result of the time evolution of normalized side mode populations for a three-frequency pump beam. We could see that side mode $m=3$ would be strongly enhanced at long time while side mode $m=1$ reduced.

\subsection{Momentum transfer in the high order forward modes}

From the above discussion we know that using multi-resonant frequencies is a promising way to get a large number of higher forward modes. When a pump laser has frequency components $\omega_{l}, \omega_{l}-8 \omega_{r}, \cdots, \omega_{l}-(n-1) \times 8 \omega_{r}$, satisfying $(n-1) \times 8 \omega_{r} \ll \omega_{l}$, with the kinetic energy of mode $m=n$ equal to $4 n^{2} \hbar \omega_{r}$, then after the condensate atoms spontaneously scattered to mode $m=1$, the end-fire mode will have frequency components $\omega_{l}-4 \omega_{r}, \omega_{l}-$ $12 \omega_{r}, \cdots, \omega_{l}-(2 n-1) \times 4 \omega_{r}$. For resonance concern, mode $m=1$ will absorb photons from the pump components $\omega_{l}, \omega_{l}-8 \omega_{r}, \cdots, \omega_{l}-(n-2) \times 8 \omega_{r}$ and emits end-fire mode photons with frequency $\omega_{l}-12 \omega_{r}, \cdots, \omega_{l}-(2 n-1) \times 4 \omega_{r}$ which are resonant with existing end-fire mode, so mode $m=2$ is produced. Like mode $m=1$, modes $m=2, m=3, \cdots, m=n-1$ can absorb pump photons and emit photons resonant to the existing end-fire mode. For example, mode $m=n-1$ will absorb photons with frequency $\omega_{l}$ and emits photons with frequency $\omega_{l}-(2 n-1) \times 4 \omega_{r}$. Therefore atoms could finally be transferred to mode $m=n$. Note that 
mode $m=n$ cannot emit resonant end-fire mode, so mode $m=n$ will be enhanced. To show it, Fig.6(b) is the simulated result of the time evolution of normalized side mode populations for a five-frequency pump beam. We could see that side mode $m=5$ would be strongly enhanced.

\subsection{Discussion}

In the experiment, to get several resonant frequencies, the laser beam from an external cavity diode laser can be split into several parts, and their frequencies are shifted individually by acoustic-optical modulators (AOMs) which are driven by phase-locked radio frequency signals, as demonstrated in the case of two resonant frequency (Bar-Gill et al., 2007; Yang et al., 2008). Therefore, the frequency difference between the beams can be controlled precisely. Furthermore, to avoid the reflection from the glass tube and formation of Bragg scattering in the experiment, the pump beam can actually deviate a few degrees from the long axis, as shown in the experiments (Fallani et al., 2005; Hilliard et al., 2008; Li, Zhou et al., 2008).

Different to the works in the configuration where the pump beam travels along the short axis of the condensate with the resonant frequency (Yang et al., 2008), where a large number of backward scattering is obvious in a two-frequency pump beam, the backward scattering is suppressed and the forward second-order mode is obviously enhanced in our case. This is due to mode competition between the forward second-order mode and the backward mode and local depletion of the superradiant process.

We have not considered the initial quantum process because its time scale is very small, shorter than $1 \mu \mathrm{s}$. In this quantum process there is also mode competition to form the end-fire modes along the long axis and suppress the emission on the other direction. This is different concept from what has been discussed above, in which case mode competition exists in the different channels satisfying the energy match and spatial condition.

For the pump beam with several resonant frequencies, not only can we obtain the high order momentum transfer which is important in the momentum manipulation for atom interferometry, but also the above analysis is useful to understand the interplay between the matter wave and light in the matter wave amplification (Kozuma, Suzuki et al., 1999; Schneble et al., 2003), atomic cooperative scattering in the optical lattice (Xu et al., 2009), and by the pump with a noisy laser (Robb \& Firth, 2007; Zhou, 2009).

\section{High order momentum states by scattering of standing wave pulses}

In this section, we apply a method for flexible manipulation of the atomic momentum states with the standing wave pulses. The atomic diffraction from standing wave pulses is demonstrated in the experiments and systematically analyzed by the band structure theory of one-dimension optical lattice. With this method, we are able to design and realize several specific momentum states, which may be applied in atomic interferometry. In principle, this method could be used for designing a wide range of possible target states.

\subsection{Theoretical model}

We consider a non-interacting condensate being diffracted by a sequence of square shaped standing wave pulses with the successive durations $\tau_{i}(i=1,2, \ldots, s+1)$, separated by the 
intervals $\tau_{f i}(i=1,2, \ldots, s)$. The standing wave consists of a pair of laser beams far-detuned enough to suppress the spontaneous emission.

The periodic potential (Denschlag et al., 2002; Morsch \& Oberthaler, 2006) introduced by the ac Stark shift can be described as $V(x)=U_{0} \cos ^{2}\left(k_{L} x\right)$, with the trap depth $U_{0}$ and the laser's wave vector $k_{L}=2 \pi / \lambda_{L}$ ( $\lambda_{L}$ is the wavelength of the laser). The lattice leads to a band structure of the energy spectra, of which the eigenvalues of the energy $E_{n, q}$ and eigenvectors $|n, q\rangle$ (Bloch states) are labeled by the quasi-momentum $q$ and the band index $n$, and they satisfy the equation:

$$
\hat{H}|n, q\rangle=E_{n, q}|n, q\rangle,
$$

where the Hamiltonian $\hat{H}=\hat{p}^{2} / 2 M+U_{0} \cos ^{2}\left(k_{L} x\right)$, with the atomic momentum $\hat{p}$ and the atomic mass $M$. The Bloch states form a quasi-momentum space. In the lattice, the spatial periodicity of the wave function results in separated peaks in momentum space, corresponding to the reciprocal lattice vector $2 k_{L}$.

When a condensate with an initial momentum $p_{m_{0}}=\hbar\left(q+2 m_{0} k_{L}\right)$ ( $\hbar$ is the Plank constant, $\left.-k_{L} \leq q \leq k_{L}, m_{0}=\ldots,-1,0,1, \ldots\right)$ is abruptly loaded into a lattice, the wave packet can be described as a superposition of the Bloch states:

$$
|\Psi(t=0)\rangle=\sum_{n=0}^{\infty}|n, q\rangle\left\langle n, q \mid p_{m_{0}}\right\rangle,
$$

where $\left\langle n, q \mid p_{m_{0}}\right\rangle=c_{n, q}\left(m_{0}\right)$. The $n^{\text {th }}$ Bloch state evolves independently as $e^{-i E_{n, q} t / \hbar}$, and the total wave function evolves as

$$
|\Psi(t)\rangle=\sum_{n=0}^{\infty} c_{n, q}\left(m_{0}\right) e^{-i E_{n, q} t / \hbar}|n, q\rangle .
$$

While the incident light is switched off after the duration $\tau_{1}$, the wave function is projected back to the momentum space from the quasi-momentum space. The coefficient $b\left(m_{0}, m, \tau_{1}\right)$ of each $\left|p_{m}\right\rangle$ state $(m=\ldots,-1,0,1, \ldots)$ can be acquainted as:

$$
b\left(m_{0}, m, \tau_{1}\right)=\sum_{n=0}^{\infty} c_{n, q}\left(m_{0}\right) c_{n, q}(m) e^{-i E_{n, q} \tau_{1} / \hbar} .
$$

For a zero initial momentum of the condensate, the subscript $q$ can be omitted for simplification and $m_{0}=0$. For one pulse scattering, the population of the $\left|p_{m}\right\rangle$ state is $P_{m}^{(1)}=\left|b\left(0, m, \tau_{1}\right)\right|^{2}$. It can be seen that the probabilities of the momentum states after one scattering pulse depend on the lattice depth and the pulse duration. The lattice depth determines the band structure and is reflected in the terms $c_{n, q}$. The pulse duration influences the phase evolution of each Bloch state as $e^{-i E_{n, q} \tau_{1} / \hbar}$.

The multi-pulse process, which consists of a number of single pulses and intervals can be solved as follows. The wave function of the condensate after the first pulse $\tau_{1}$ can be derived from Eq. (8) as

$$
\left|\Psi\left(\tau_{1}, t\right)\right\rangle=\sum_{m} b\left(m_{0}, m, \tau_{1}\right) e^{-i E^{(m)} t / \hbar}\left|2 m \hbar k_{L}\right\rangle
$$


After the first interval $\tau_{f 1}$ and the second pulse $\tau_{2}$, the population of the $\left|p_{m}\right\rangle$ state can be achieved as

$$
P_{m}^{(2)}=\left|\sum_{m_{1}} b\left(m_{0}, m_{1}, \tau_{1}\right) e^{-i E^{\left(m_{1}\right)} \tau_{f 1} / \hbar} b\left(m_{1}, m, \tau_{2}\right)\right|^{2} .
$$

As shown in Eq. (10), the population is affected by the two pulses, the first one corresponding to $b\left(m_{0}, m_{1}, \tau_{1}\right)$, and the second one corresponding to $b\left(m_{1}, m, \tau_{2}\right)$. During the interval $\tau_{f 1}$, the phase of the $\left|p_{m_{1}}\right\rangle$ state evolves along the time as $e^{-i E^{\left(m_{1}\right)} t / \hbar}$, where $E^{\left(m_{1}\right)}=\left(2 m_{1} \hbar k_{L}\right)^{2} / 2 M=$ $4 m_{1}^{2} E_{R}$ is the kinetic energy, and $E_{R}=\left(\hbar k_{L}\right)^{2} / 2 M$ is the single photon recoil energy. The interval $\tau_{f 1}$ produces a phase shift $e^{-i E^{(m)} \tau_{f 1} / \hbar}$ and contributes to the momentum distribution. In the same way, the population of the $\left|p_{m}\right\rangle$ state after $(s+1)$ pulses can be achieved as:

$$
P_{m}^{(s+1)}=\left|\sum_{m_{1}, m_{2}, \cdots, m_{s}} \prod_{i=1}^{s+1} b\left(m_{i-1}, m_{i}, \tau_{i}\right) \prod_{i=1}^{s} e^{-i E^{\left(m_{i}\right)} \tau_{f_{i}} / \hbar}\right|^{2},
$$

with $m_{0}=0$, and $m_{s+1}=m$.

From the analysis above, the momentum distribution after a sequence of pulses' scattering is influenced by not only the lattice pulses with the term $\prod_{i=1}^{s+1} b\left(m_{i-1}, m_{i}, \tau_{i}\right)$, but also the intervals among the pulses as reflected in the term $\prod_{i=1}^{s} e^{-i E^{\left(m_{i}\right)} \tau_{f_{i}} / \hbar}$. Although the populations of the momentum states do not change during the intervals, the phase-evolution rates of the momentum states with different kinetic energies are not identical. The phase deviations between the states oscillate from 0 to $2 \pi$ with the interval, and the heterogeneously accumulated phases change the distribution of the condensate in the quasi-momentum space.

\subsection{Experiments of standing wave pulse sequences}

We performed the experiments of a condensate in a magnetic trap (MT) (see Fig. 7(a)) being scattered by a sequence of standing wave pulses (see Fig. 7(b)). As shown in Fig. 7(c), after pre-cooling, a cigar shaped ${ }^{87} \mathrm{Rb}$ condensate of $2 \times 10^{5}$ atoms in $5^{2} S_{1 / 2}\left|F=2, M_{F}=2\right\rangle$ state was achieved by the radio frequency (RF) cooling in the magnetic trap, of which the axial frequency is $20 \mathrm{~Hz}$ and the radial frequency is $220 \mathrm{~Hz}$ (Yang et al., 2008; Zhou et al., 2010). A pair of counter-propagating laser beams, of which the durations were controlled by an acousto-optical modulator, and the amplitudes were adjusted by the injection current of a tapered-amplifier, were applied to the condensate along the axial direction. The linear polarized incident light at the wavelength $\lambda_{L}=852 \mathrm{~nm}$ was focused with a waist of $110 \mu \mathrm{m}$ to cover the condensate. The trap depth, which was calibrated by Kapitza-Dirac scattering experimentally, reached $120 E_{R}$, corresponding to the light power of $320 \mathrm{~mW}$. The incident light and the magnetic trap were simultaneously shut after the BEC-light interaction. After $30 \mathrm{~ms}$ free falling and ballistic expansion, the atomic gas was pictured by absorption imaging. Since the minimum gap between different momentum states is $2 \hbar k$, which is much larger than the momentum width of a single momentum state, the components with different momenta will be separated in the TOF (time of flight) signal (see Fig. 7(d)), and the atomic number of each momentum state is possible to be read separately as $N_{m}$ (the momentum 
(a)

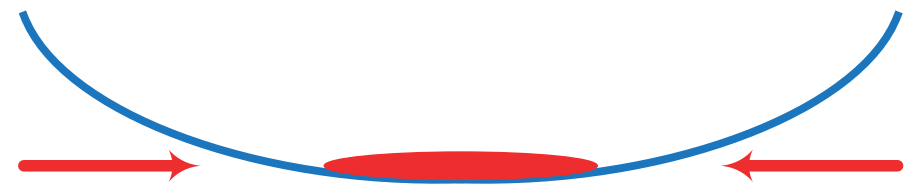

(b)

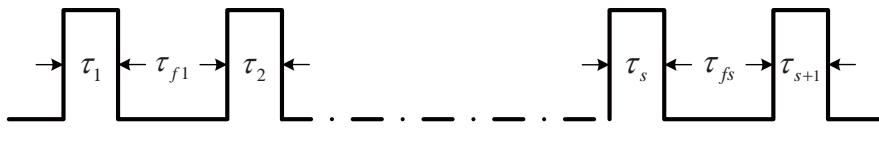

(c)

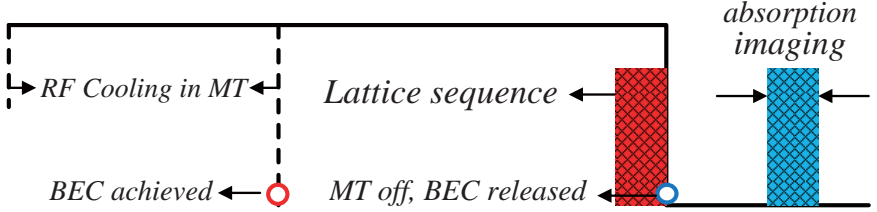

(d)

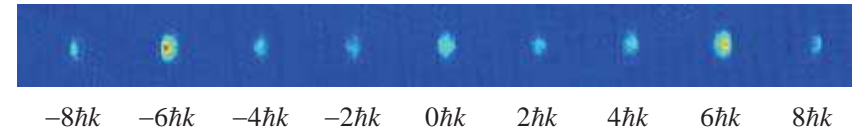

Fig. 7. (Color online) (a) A pair of counter-propagating light beams are applied to a condensate in magnetic trap. (b) The scattering process consists of a sequence of standing wave pulses, which contained $s$ intervals with widths $\tau_{f i}(i=1,2, \ldots s)$ and $s+1$ light pulses with durations $\tau_{i}(\mathrm{i}=1,2, \ldots s+1)$. The incident light's wavelength is $852 \mathrm{~nm}$ and its maximum intensity can reach $120 E_{R}$. (c) The procedure for the experiments is shown. The condensate is exposed to a sequence of standing wave pulses and then released from the magnetic trap. The absorption images of the condensate can be observed after the free falling. (d) A TOF signal obtained in our experiment.

order $m=\ldots,-2,-1,0,1,2)$. The relative population of the momentum state $|2 m \hbar k\rangle$ can be evaluated as $N_{m} / \sum N_{m}$.

The lattice in our experiments is quite deep, so we concentrate on the short-pulse diffractions to avoid the de-coherence and heating effects of long pulses relevant for Bragg scattering. However, for more flexible momentum manipulation, our pulses are not so short as the Raman-Nath pulses (Huckans et al., 2009) used in previous works.

A brief introduction to the Raman-Nath regime is given in the following for comparison. In the scattering process, the evolution during the free evolution intervals is analyzed as in previous section, while the effect of lattice with adequately short duration $\tau$ can be analytically solved by the Schrödinger equation $i \hbar \partial|\Psi(t)\rangle / \partial t=\hat{H}|\Psi(t)\rangle$, after omitting the atomic kinetic energy term $\hat{p}^{2} / 2 M$ in the Hamiltonian. This approximation can be made while the displacement of the scattered atoms during the interaction time is much smaller than the spatial period of the standing wave. Equivalently, the standing wave duration $\tau$ and the single photon recoil frequency $\omega_{r}=\hbar k_{L}^{2} / 2 M$ have to fulfill $\tau \ll 1 / \omega_{r}$. The pulse is able to split a stationary condensate into components with symmetrical momenta $p_{n}=2 n \hbar k_{L}(n=0, \pm 1, \pm 2, \ldots)$, with corresponding populations $P_{n}=J_{n}^{2}\left(U_{0} \tau / 2 \hbar\right)$, where $J_{n}(z)$ are Bessel functions of the first kind. 


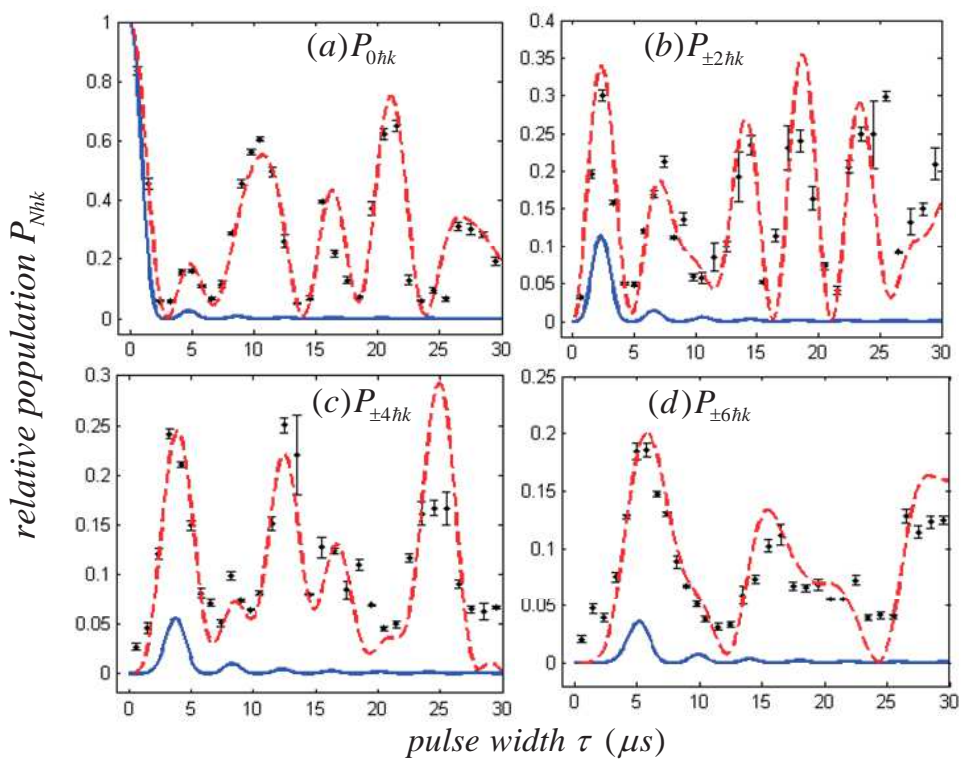

Fig. 8. (Color online) Single pulse scattering of condensate: The black dots represent the experiment results. The blue solid line is the theoretical analysis with Raman-Nath approximation. The red dashed line is the numerical simulation with band structure theory. Figure (a), (b), (c) and (d) correspond to the relative populations of the condensates with the momenta $0 \hbar k, \pm 2 \hbar k, \pm 4 \hbar k$ and $\pm 6 \hbar k$ respectively.

First we demonstrate a one-pulse scattering experiment. A condensate is exposed to a standing wave pulse with depth $100 E_{R}$ and duration varying from 0 to $30 \mu \mathrm{s}$. The relative populations of the condensates with the momenta $0 \hbar k, \pm 2 \hbar k, \pm 4 \hbar k$ and $\pm 6 \hbar k$, corresponding to Fig. 8(a), (b), (c) and (d) respectively, are measured and theoretically analyzed. In addition, the theoretical analysis with the Raman-Nath approximation, is also shown in the figure for comparison. It can be seen that within $3 \mu$ s the theoretical analysis with the Raman-Nath approximation (blue solid line) is close to the experimental results (black dots), and so is the theoretical analysis with band structure theory (red dashed line). When the pulse duration exceeds $3 \mu$ s, the analysis with the Raman-Nath approximation gradually goes far away from the experimental results, while the numerical simulation with band structure theory still agrees with the experimental results along the entire time scale. As shown in Fig. 8, the probability of each momentum state oscillates with the pulse duration as described by the band structure theory. It is clear that, in the single pulse scattering process, the band structure theory works well not only for the short pulse but also for the longer pulse, because the atomic motion has been taken into account. So the atomic diffraction by a single standing wave pulse can be predicted in a wider range of pulse duration with the band structure theory.

Then we increase the number of pulses in the experiments to explore the extra factors influencing the momentum distributions. In every sequence, all the pulses are the same and all the intervals are identical to make the experiments more convenient to carry out. For further 
comparison between the band structure theory and the analysis in the Raman-Nath regime, every single pulse is made short enough for the Raman-Nath approximation.
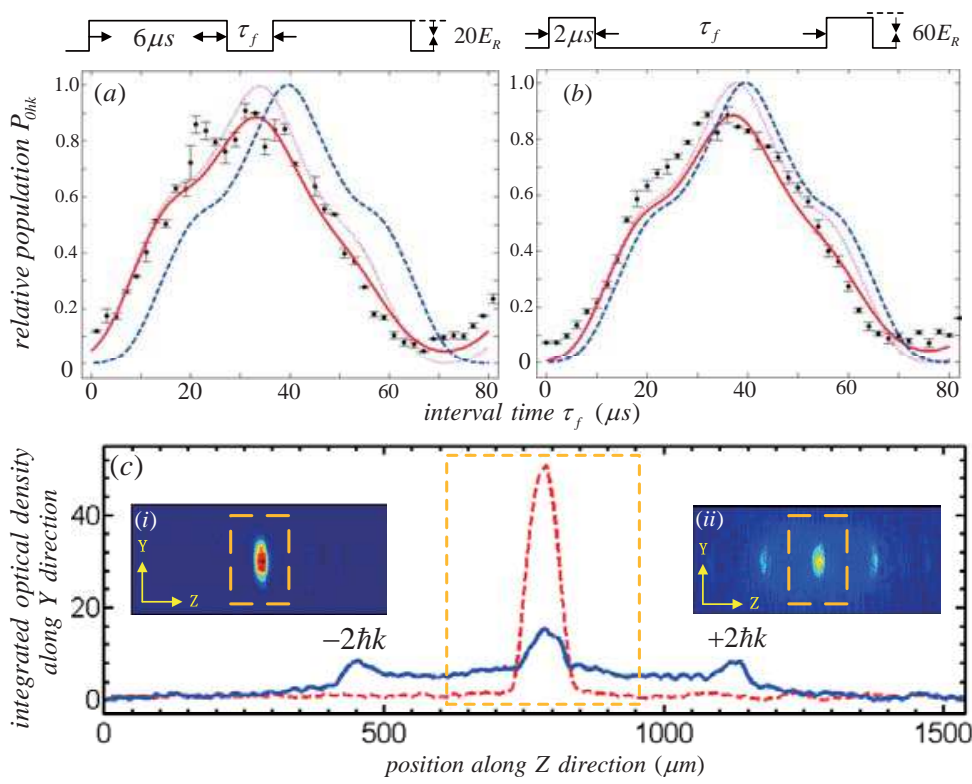

Fig. 9. (Color online) Two-pulse scattering of the condensate: The relative populations of the stationary condensate $P_{0 \hbar k}$ versus the varied intervals $\tau_{f}$ is shown. The parameters of the experiments are described above each figure. The black dots are the experimental results. The blue dashed line is the analysis with Raman-Nath approximation. The magenta dotted line is the numerical simulation with band structure theory. The red solid line is a numerical simulation taking into account the momentum dispersion. (c) A demonstration of momentum expansion introduced by s-wave scattering. The TOF signal (i) corresponds to the dashed curve and pictures the momentum distribution before the former lattice pulse. The signal (ii) corresponds to the solid curve and shows the momentum distribution after the former pulse. The momentum width along $\mathrm{Z}$ direction (the lattice direction) is evaluated based on the parts in the dashed boxes which include the momenta from $-\hbar k$ to $\hbar k$.

Two experiments of two-pulse scattering are demonstrated in Fig. 9, in which the relative populations of the stationary condensate $P_{0 \hbar k}$ versus the varied intervals $\tau$ is shown. The parameters of the scattering pulses used in different sequences are chosen to be of the same products of the lattice depth and the pulse duration, so that each pulse affects the condensate equivalently. As shown in the figure, the intervals actually affect the final momentum distribution, and the theoretical analysis with the band structure theory and Raman-Nath approximation both picture well the evolution of the atomic distributions versus the interval between the two pulses. The results of two-pulse scattering can be explained as the fact that since the phase shift accumulated during the interval varies harmonically from 0 to $2 \pi$, the probability of the stationary condensate oscillates between the minimum and the maximum. When the phase shift is $2 \pi$ with the interval $\pi \hbar / 2 E_{R}$ (around $80 \mu s$ ), the wave function is little affected by the interval and the two pulses diffract the condensate as one combined 
pulse to make the probability $P_{0 \hbar k}$ the minimum. While the phase shift is $\pi$ with the interval $\pi \hbar / 4 E_{R}$ (about $40 \mu s$ ), the second pulse produces an effect opposite of the first one and diffract the non-stationary components of the condensate back to the stationary one and make the probability $P_{0 \hbar k}$ the maximum.

It also can be acquainted from Fig. 9 that the numerical simulation with band structure theory is much closer to the experimental results than the analytical solution with Raman-Nath approximation. It is conjectured that the phase evolution during the scattering process makes the difference. The phase evolution in the scattering process is neglected in the Raman-Nath approximation, but not in the numerical simulation with band structure theory. Although the duration of the scattering process is short, the phase shifts in the scattering process still increase. The phase shift in the scattering process needs to be taken into account and influences the final momentum distribution. As a result, the longer the scattering pulse is, the larger the difference is. Although the maximum of the probability $P_{0 \hbar k}$ corresponds to the interval $\pi \hbar / 4 E_{R}$, the two-pulse experiments in Fig. 9 can clearly show that the longer pulse leads to the larger difference. In Fig. 9 (a), the pulse duration is $6 \mu s$, the probability $P_{0 \hbar k}$ reaches the maximum with the interval $34 \mu s$. In Fig. $9(\mathrm{~b})$, the pulse duration is $2 \mu s$, the probability $P_{0 \hbar k}$ gets to the top with the interval $38 \mu \mathrm{s}$.

Nevertheless, there is still some obvious deviation between the simulation and the experimental results. It is observed that the momentum width has been expanded after the former pulse (see Fig. 9(c)), because of the s-wave scattering between the different momentum states. Consequently, this dispersion process is approximated to an initial momentum width of $\sim 0.1 \hbar k_{L}$ on average to optimize the numerical simulation. Unlike the analysis without momentum width, phase evolution is different for different initial momenta and results in a phase dispersion. The quasi modes obtained at the end of the diffraction process result from the linear superposition of final states obtained after time evolutions of the different momenta populated the initial BEC. It can be seen from Fig. 9 that the approximation is effective.

As discussed in (Li, Deng et al., 2008), the maximum of the probability $P_{0 \hbar k}$ will never reach 1 thanks to the imperfect optical lattice. In our case, the momentum expansion is an explanation of the similar situation as shown in Fig. 9. Since the momentum width is considered, the phase shift is populated around $\pi$ with a width, instead of a definite $\pi$, with the interval $\pi \hbar / 4 E_{R}$. In other words, there is no interval that accumulates a phase shift exactly equal to $\pi$, so with any interval, the second standing wave pulse is not able to diffract all the condensates back to the stationary part.

\subsection{Manipulate the momentum states as design}

The experiments and the numerical simulations above have shown the possibility and feasibility of the manipulation of a condensate's momentum states. We manage to design several two-pulse sequences to achieve high contrast momentum states such as $| \pm 2 \hbar k\rangle$, $| \pm 4 \hbar k\rangle$ and $| \pm 6 \hbar k\rangle$, which may be useful in atomic interferometry (Beattie et al., 2009; Rohwedder, 2001). For each state, we apply two totally different two-pulse sequences to show the flexibility of the method. The general method to achieve the target states is to find out the condition of the minimum of the square deviation $\Delta^{2}=\sum_{m=-\infty}^{+\infty}\left(P_{m}^{g}-P_{m}\right)^{2}$, where $P_{m}^{g}$ is the probability of $\left|2 m \hbar k_{L}\right\rangle$ in the goal state, and $P_{m}$ is that generated by the sequence. A 
second method, as the target is to obtain the highest population of some certain momentum state, consists in scanning the set of initial conditions and choose the one corresponding to the maximum value of the desired population. We apply the two methods above separately and obtain the same pulse sequences. As shown in Fig. 10, the experimental results (the black

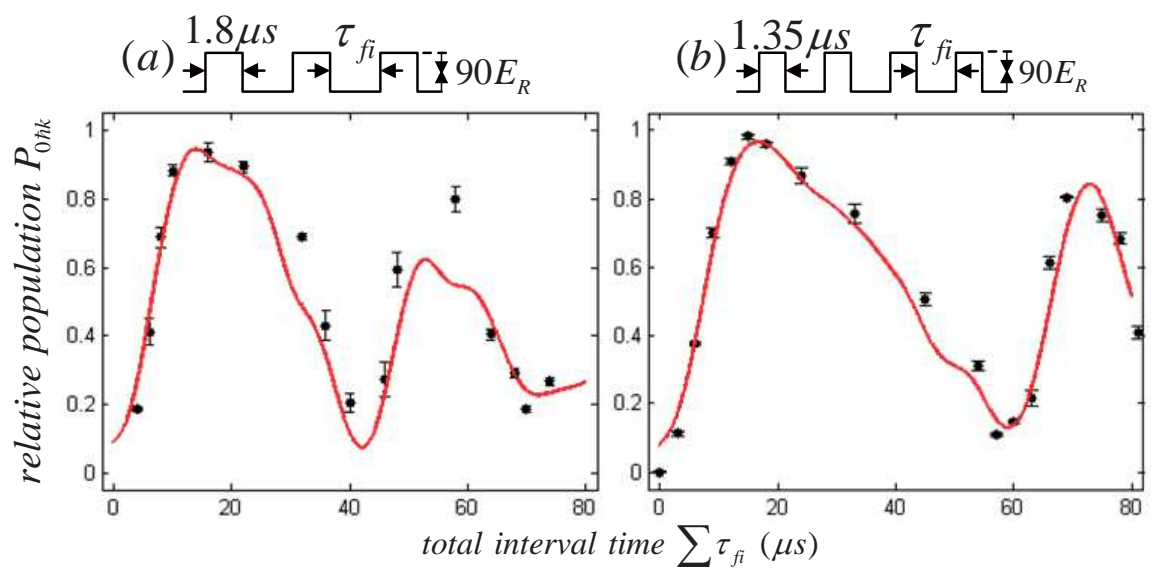

Fig. 10. Experimental realization of designed momentum states. The expected momentum state is $\pm 2 \hbar k_{L}\left((\mathrm{a})\right.$ and (b)), $\pm 4 \hbar k_{L}\left((\mathrm{c})\right.$ and (d)) and $\pm 6 \hbar k_{L}((\mathrm{e})$ and (f)). The pulse sequences are shown above each figure. The black round dots are experimental results. The blue diamond dots are the expectations based on the design. The red square dots are the modified design with momentum width, which agree with the experiments better.

round dots) agree well with the expectations of the designs (the blue diamond dots), whether the pulses are in the Raman-Nath regime (see Fig. 10(b)) or not (see others in Fig. 10). When the momentum dispersion is being considered, the expected momentum distributions (the red square dots) get closer to the experiments, where the figures only display the relative populations of the target states and omit the others for the figures being more clear.

It can be seen from Fig. 10 that the momentum width correction can improve the precision of the prediction with our method. The average relative deviation between the experimental results and the expected values without including the momentum width is $25.03 \%$, while the deviation is decreased to $13.15 \%$ with the correction.

An asymmetry of the momenta can be observed in Fig. 10, and it may ascribe to the following factors. Besides the measurement error, there is an imperfection of the standing wave, brought forth by the unbalanced intensity of the laser beams. External field (such as the magnetic trap) fluctuations during the scattering process may also affect the momentum distribution.

\subsection{Discussion}

The band structure theory is a global method to deal with the standing wave scattering a condensate, while Bragg and Raman-Nath scattering are two special situations which can be analytically solved with their respective approximations. In the Bragg regime, the potential height introduced by the standing wave is restrained below $4 E_{R}$ and that leads to the difficulty 
of generating higher order momentum states. In the Raman-Nath regime, the intensity of the standing wave is not limited so that higher order momentum states can be generated symmetrically (Gadway et al., 2009; Sapiro et al., 2009b). However the pulse duration has to be short enough to neglect the atomic motion, so the momentum states can not be predicted in this regime if the pulse duration is slightly longer. In our work, the scattering can be well explained and numerically analyzed within a much wider range of pulse intensity and duration. So it is natural that more momentum states can be generated.

Some valuable states, such as $| \pm 2 \hbar k\rangle,| \pm 4 \hbar k\rangle$ and $| \pm 6 \hbar k\rangle$ states with high contrast, can not be realized by the single pulse scattering, while they can be realized by a sequence of standing wave pulses. A sequence of lattice pulses is a more effective and flexible tool for momentum manipulation. It can generate many useful momentum states, in addition to the ones demonstrated in our work.

Although the numerical simulation is corrected to take into account the momentum dispersion, some deviations between the experiments and the simulation still exist. The inaccuracy of the lattice-depth calibration, which is $5 \%$ at least, is one of the reasons. The phase shift introduced by the magnetic trap is another one, while the influence is estimated to be within $0.03 \%$, which is below the experimental uncertainty. The heating and momenta exchange during the s-wave scattering may also lead to some differences.

\section{Conclusion}

We have shown in this chapter two methods of getting high order momentum states by resonant superradiant scattering and by a sequences of pulsed standing waves, corresponding to traveling wave scattering and standing wave scattering, respectively. Different to Bragg scattering, we can get a large number of high-order forward modes by resonant frequency components of the pump beam by the former method through the mode competition between the different resonant channels and the local depletion of the spatial distribution. While with the latter method a symmetric momentum distribution is generated, with one pair of momentum states designed to be mostly populated. Both methods are beneficial to a lager momentum transfer in atom manipulation for the atom interferometry and atomic optics.

\section{References}

Bar-Gill, N., Rowen, E. E. \& Davidson, N. (2007). Spectroscopy of strong-pulse superradiance in a Bose-Einstein condensate, Phys. Rev. A 76: 043603.

Baumgärtner, F., Sewell, R. J., Eriksson, S., Llorente-Garcia, I., Dingjan, J., Cotter, J. P. \& Hinds, E. A. (2010). Measuring energy differences by BEC interferometry on a chip, Phys. Rev. Lett. 105: 243003.

Beattie, S., Barrett, B., Chan, I., Mok, C., Yavin, I. \& Kumarakrishnan, A. (2009). Atom-interferometric studies of light scattering, Phys. Rev. A 80: 013618.

Cola, M. M., Volpe, L. \& Piovella, N. (2009). Accelerated superradiance and collective atomic recoil lasing with a two-frequency pump, Phys. Rev. A 79: 013613.

Cronin, A. D., Schmiedmayer, J. \& Pritchard, D. E. (2009). Optics and interferometry with atoms and molecules, Rev. Mod. Phys. 81: 1051. 
Deng, L., Hagley, E. W., Denschlag, J., Simsarian, J. E., Edwards, M., Clark, C. W., Helmerson, K., Rolston, S. L. \& Phillips, W. D. (1999). Temporal matter-mave-dispersion Talbot effect, Phys. Rev. Lett. 83: 5407.

Denschlag, J. H., Simsarian, J. E., Häffner, H., McKenzie, C., Browaeys, A., Cho, D., Helmerson, K., Rolston, S. L. \& Phillips, W. D. (2002). A Bose-Einstein condensate in an optical lattice, Journal of Physics B: Atomic, Molecular and Optical Physics 35: 3095.

Edwards, M., Benton, B., Heward, J. \& Clark, C. W. (2010). Momentum-space engineering of gaseous Bose-Einstein condensates, Phys. Rev. A 82: 063613.

Fallani, L., Fort, C., Piovella, N., Cola, M., Cataliotti, F. S., Inguscio, M. \& Bonifacio, R. (2005). Collective atomic recoil in a moving Bose-Einstein condensate: From superradiance to Bragg scattering, Phys. Rev. A 71: 033612.

Gadway, B., Pertot, D., Reimann, R., Cohen, M. G. \& Schneble, D. (2009). Analysis of Kapitza-Dirac diffraction patterns beyond the Raman-Nath regime, Opt. Express 17: 19173.

Guo, R., Zhou, X. \& Chen, X. (2008). Enhancement of motional entanglement of cold atoms by pairwise scattering of photons, Phys. Rev. A 78: 052107.

Gupta, S., Leanhardt, A. E., Cronin, A. D. \& Pritchard, D. E. (2001). Coherent manipulation of atoms with standing light waves, Comptes Rendus de l'Acadlęmie des Sciences - Series IV - Physics 2: 479.

Hilliard, A., Kaminski, F., le Targat, R., Olausson, C., Polzik, E. S. \& Müller, J. H. (2008). Rayleigh superradiance and dynamic Bragg gratings in an end-pumped Bose-Einstein condensate, Phys. Rev. A 78: 051403.

Huckans, J. H., Spielman, I. B., Tolra, B. L., Phillips, W. D. \& Porto, J. V. (2009). Quantum and classical dynamics of a Bose-Einstein condensate in a large-period optical lattice, Phys. Rev. A 80: 043609.

Inouye, S., Chikkatur, A. P., Stamper-Kurn, D. M., Stenger, J., Pritchard, D. E. \& Ketterle, W. (1999). Superradiant Rayleigh scattering from a Bose-Einstein condensate, Science 285: 571.

Keller, C., Schmiedmayer, J., Zeilinger, A., Nonn, T., DÍźrr, S. \& Rempe, G. (1999). Adiabatic following in standing-wave diffraction of atoms, Applied Physics B: Lasers and Optics 69: 303.

Kozuma, M., Deng, L., Hagley, E. W., Wen, J., Lutwak, R., Helmerson, K., Rolston, S. L. \& Phillips, W. D. (1999). Coherent splitting of Bose-Einstein condensed atoms with optically induced Bragg diffraction, Phys. Rev. Lett. 82: 871.

Kozuma, M., Suzuki, Y., Torii, Y., Sugiura, T., Kuga, b.-g. T., Hagley, E. W. \& Deng, L. (1999). Phase-coherent amplification of matter waves, Science 286: 2309.

Li, J., Zhou, X., Yang, F. \& Chen, X. (2008). Superradiant Rayleigh scattering from a Bose-Einstein condensate with the incident laser along the long axis, Phy. Lett. A 372: 4750 .

Li, K., Deng, L., Hagley, E. W., Payne, M. G. \& Zhan, M. S. (2008). Matter-wave self-imaging by atomic center-of-mass motion induced interference, Phys. Rev. Lett. 101: 250401.

Meystre, P. (2001). Atom optics, Springer series on atomic, optical, and plasma physics, AIP Press/Springer.

Moore, M. G. \& Meystre, P. (1999). Theory of superradiant scattering of laser light from Bose-Einstein condensates, Phys. Rev. Lett. 83: 5202. 
Morsch, O. \& Oberthaler, M. (2006). Dynamics of Bose-Einstein condensates in optical lattices, Rev. Mod. Phys. 78: 179.

Ovchinnikov, Y. B., Müller, J. H., Doery, M. R., Vredenbregt, E. J. D., Helmerson, K., Rolston, S. L. \& Phillips, W. D. (1999). Diffraction of a released Bose-Einstein condensate by a pulsed standing light wave, Phys. Rev. Lett. 83: 284.

Piovella, N., Beretta, V., Robb, G. R. M. \& Bonifacio, R. (2003). Photon echo in the superradiant light scattering from a Bose-Einstein condensate, Phys. Rev. A 68: 021801.

Pritchard, D. E., Cronin, A. D., Gupta, S. \& Kokorowski, D. A. (2001). Atom optics: old ideas, current technology, and new results, Ann. Phys. 10: 35.

$\mathrm{Pu}, \mathrm{H} .$, Zhang, W. \& Meystre, P. (2003). Wave mixing of optical pulses and Bose-Einstein condensates, Phys. Rev. Lett. 91: 150407.

Robb, G. R. M. \& Firth, W. J. (2007). Collective atomic recoil lasing with a partially coherent pump, Phys. Rev. Lett. 99: 253601.

Rohwedder, B. (2001). Multiple atomic wave interferometry with standing-waves of light, The European Physical Journal D - Atomic, Molecular, Optical and Plasma Physics 13: 255.

Sadler, L. E., Higbie, J. M., Leslie, S. R., Vengalattore, M. \& Stamper-Kurn, D. M. (2007). Coherence-enhanced imaging of a degenerate Bose-Einstein gas, Phys. Rev. Lett. 98: 110401.

Sapiro, R. E., Zhang, R. \& Raithel, G. (2009a). Atom interferometry using Kapitza-Dirac scattering in a magnetic trap, Phys. Rev. A 79: 043630.

Sapiro, R. E., Zhang, R. \& Raithel, G. (2009b). Reversible loss of superfluidity of a Bose-Einstein condensate in a 1D optical lattice, New Journal Of Physics 11: 013013.

Schneble, D., Torii, Y., Boyd, M., Streed, E. W., Pritchard, D. E. \& Ketterle, W. (2003). The onset of matter-wave amplification in a superradiant Bose-Einstein condensate, Science 300: 475.

Slama, S., Krenz, G., Bux, S., Zimmermann, C. \& Courteille, P. W. (2007). Cavity-enhanced superradiant Rayleigh scattering with ultracold and Bose-Einstein condensed atoms, Phys. Rev. A 75: 063620.

Torii, Y., Suzuki, Y., Kozuma, M., Sugiura, T., Kuga, T., Deng, L. \& Hagley, E. W. (2000). Mach-Zehnder Bragg interferometer for a Bose-Einstein condensate, Phys. Rev. A 61: 041602.

Wu, S., Wang, Y.-J., Diot, Q. \& Prentiss, M. (2005). Splitting matter waves using an optimized standing-wave light-pulse sequence, Phys. Rev. A 71: 043602.

Xiong, W., Yue, X., Wang, Z., Zhou, X. \& Chen, X. (2011). Manipulating the momentum state of a condensate by sequences of standing-wave pulses, Phys. Rev. A 84: 043616.

Xu, X., Zhou, X. \& Chen, X. (2009). Spectroscopy of superradiant scattering from an array of Bose-Einstein condensates, Phys. Rev. A 79: 033605.

Yang, F., Zhou, X., Li, J., Chen, Y., Xia, L. \& Chen, X. (2008). Resonant sequential scattering in two-frequency-pumping superradiance from a Bose-Einstein condensate, Phys. Rev. A 78: 043611.

Zhou, X. (2009). Cooperative atomic scattering of light from a laser with a colored noise spectrum, Phys. Rev. A 80: 023818.

Zhou, X., Fu, J. \& Chen, X. (2009). High-order momentum modes by resonant superradiant scattering, Phys. Rev. A 80: 063608.

Zhou, X., Yang, F., Yue, X., Vogt, T. \& Chen, X. (2010). Imprinting light phase on matter-wave gratings in superradiance scattering, Phys. Rev. A 81: 0136151. 
Zobay, O. \& Nikolopoulos, G. (2007). Sequential superradiant scattering from atomic Bose-Einstein condensates, Laser Physics 17: 180.

Zobay, O. \& Nikolopoulos, G. M. (2006). Spatial effects in superradiant Rayleigh scattering from Bose-Einstein condensates, Phys. Rev. A 73: 013620. 


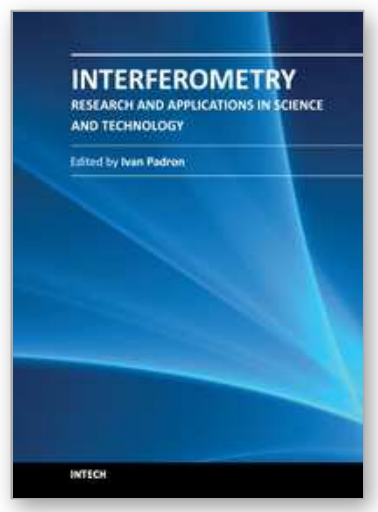

\section{Interferometry - Research and Applications in Science and Technology}

Edited by Dr Ivan Padron

ISBN 978-953-51-0403-2

Hard cover, 462 pages

Publisher InTech

Published online 21, March, 2012

Published in print edition March, 2012

This book provides the most recent studies on interferometry and its applications in science and technology. It is an outline of theoretical and experimental aspects of interferometry and their applications. The book is divided in two sections. The first one is an overview of different interferometry techniques and their general applications, while the second section is devoted to more specific interferometry applications comprising from interferometry for magnetic fusion plasmas to interferometry in wireless networks. The book is an excellent reference of current interferometry applications in science and technology. It offers the opportunity to increase our knowledge about interferometry and encourage researchers in development of new applications.

\section{How to reference}

In order to correctly reference this scholarly work, feel free to copy and paste the following:

Xiaoji Zhou and Xuguang Yue (2012). High Order Momentum States by Light Wave Scattering, Interferometry - Research and Applications in Science and Technology, Dr Ivan Padron (Ed.), ISBN: 978-953-51-0403-2, InTech, Available from: http://www.intechopen.com/books/interferometry-research-and-applications-in-scienceand-technology/high-order-momentum-states-by-light-wave-scattering

\section{INTECH}

open science | open minds

\section{InTech Europe}

University Campus STeP Ri

Slavka Krautzeka 83/A

51000 Rijeka, Croatia

Phone: +385 (51) 770447

Fax: +385 (51) 686166

www.intechopen.com

\section{InTech China}

Unit 405, Office Block, Hotel Equatorial Shanghai

No.65, Yan An Road (West), Shanghai, 200040, China

中国上海市延安西路65号上海国际贵都大饭店办公楼405单元

Phone: +86-21-62489820

Fax: +86-21-62489821 
(C) 2012 The Author(s). Licensee IntechOpen. This is an open access article distributed under the terms of the Creative Commons Attribution 3.0 License, which permits unrestricted use, distribution, and reproduction in any medium, provided the original work is properly cited. 